\title{
Robot Motion Generation by Hand Demonstration
}

\author{
Sakmongkon Chumkamon, Umaporn Yokkampon, Eiji Hayashi, Ryusuke Fujisawa \\ School of Computer Science and Systems Engineering, Kyushu Institute of Technology, Japan \\ E-mail:m-san@mmcs.mse.kyuetech.ac.jp,may@mmcs.mse.kyuetech.ac.jp,haya@mse.kyutech.ac.jp, \\ fujisawa@ces.kyutech.ac.jp
}

\begin{abstract}
Since traditional robot teaching requires time and instruction to the robot motion, we present a systematic framework based on deep learning and experiment for generating robot motion trajectories from human hand demonstration. In this system, the worker could teach robot easier rather than assigning the instruction to the robot controller manually. Therefore, the robot can imitate the action in a new situation instead of directly teaching the robot arm. Our contributions include three points 1) the real-time extracting method of hand movement without marker using hand detection in 3D from human 2) the motion generalization of the hand trajectories from human 3) Robot path planning for grasping and place the object to the target. We also present the experiment conducted by the user movement for real data and evaluate the system using the manipulator robot. The investigation shows the pick-and-place task of the robot for food by hand demonstration.
\end{abstract}

Keywords: List four to six keywords which characterize the article.

\section{Introduction}

In order to push country economic, smart automation in the industrial sector is critical to improving nowadays. Since the merit of artificial intelligence research could facilitate various tasks in industrial. Therefore, we can develop a factory that could produce products faster with higher precision. In a smart factory, the robot is essential to assembly because it is more reliable and accurate than human.

The manipulator robot actually is controlled by joint position; however, we usually control the robot in Cartesian space; therefore, we have to calculate the inverse kinematic (IK). One of IK that is popular recently is Trac-IK and KDL, which can figure the solution more than traditional methods ${ }^{1}$. The robot also needs to plan the trajectory according to the environments such as avoiding the collision or moving to the target. The recent motion planning method is Open Motion Planning Library which is popular, reliable and fast for solving the trajectory motion in various movement such as robot arm motion, vehicle, drone ${ }^{2}$.
In robot learning by demonstration, recent research develops the robot to predict and learning the motion from a human. However, the research develops from the device which is attached to the human body to extracting the information of motion ${ }^{3}$. Additionally, some research develops the teleoperation to the robot to learn how to move following the human motion ${ }^{4}$.

In this paper, we propose and contribute the robot system that can generate the robotics motion from human hand demonstration and generalize the hand motion by Dynamic Movement Primitive (DMP) ${ }^{5}$. Then we apply the motion to the robot. Besides, this system uses TracIK for kinematic and OMPL for path planning after DMP process. Fig. 1 shows the overview of our proposed, which consists of hand demonstration with grasping object and perception using RGBD cameras with hand position estimation then robot motion using the manipulator and visualization using PCs.

For the rest of the paper, section 2 presents the framework of robotic motion generation approach by human demonstration. Section 3 explains the experiments as well as evaluation of the robotics motion. Finally, we 


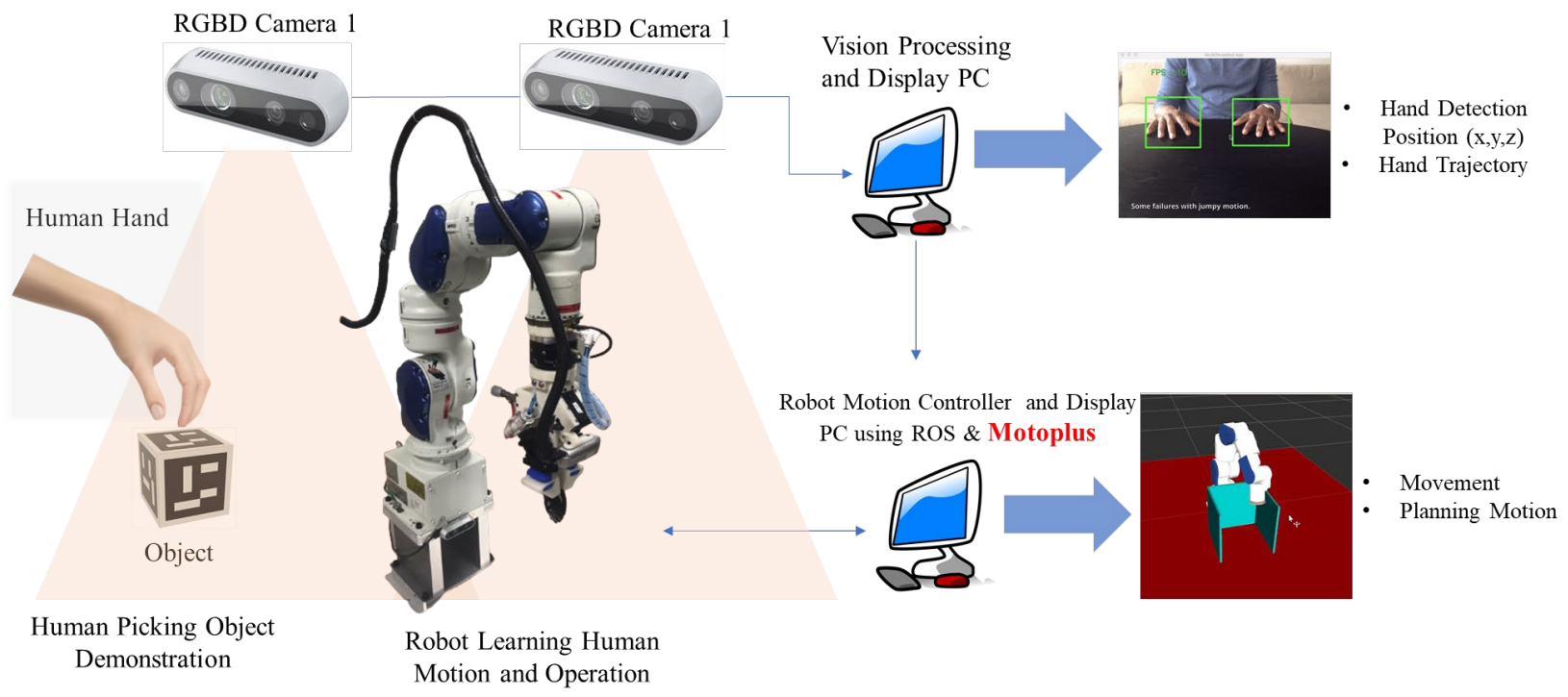

Fig. 1. The overview system of the Robot Motion Generation by Hand Demonstration.

present the conclusion, discussion and critical finding in this paper.

\section{Approach}

In this section, we explain the overview system including robot hardware configuration, hand recognition, hand position and trajectory determining. Finally, the motion repeating following the human hand demonstration.

\subsection{Hand Position Extraction}

Firstly, we implement the method to collect the data from the demonstration by record human hand motion. We divide the process into two steps. The First step is hand detection. We introduce the deep learning-based model which is Single Shot Detection (SSD) for real-time hand detection from a colour image ${ }^{6}$. SSD could outcome the detection box of hand area then we apply the human skin filter to getting only hand region. Later, we determine the hand position by calculating the centroid of the hand region. Therefore, we could get the hand position in 2dimension. Since we use the RGBD camera, we apply both colour and depth image to map each other. After that, we map the $2 \mathrm{D}$ hand position into depth image to get the hand position in 3-dimension cartesian coordinate. Finally, we concatenate the point while the human moves to be the trajectory of hand motion and record to send to the robot.

\subsection{Robot Trajectory Generation and Motion}

Since the robot knew the hand motion trajectory, we can then plan the robot's motion according to the workspace constraint and the trajectory. Firstly, we set the home pose to initialize the robot joints to prepare a starting position to move following the trajectory. When the robot gets the new trajectory from demonstration, it will plan motion using the Open Motion Planning Library (OMPL) and Trac-IK inverse kinematic. In this implementation, we utilize the MoveIt tool with Robotics Operation System to organize motion planning and collision protection among the workspace environment. After complete recording the trajectory, then the robot moves following the human hand trajectory.

\subsection{Experiment Setup}

We use the industrial robot with 7 degrees of freedom and soft gripper tool of end-effector for the hardware configuration system since we aim our robot to grasp various food. For robot control system is implemented in the desktop PC with GPU Nvidia RTX 2080Ti. The PC also operates the SSD for hand detection, archiving the average hand detection 55 frame per second. In experiment setup, the robot is mounted on the robot base station with the camera mounted at the base of the robot. We then calibrate the camera position translation to the robot position and orientation.

(C) The 2021 International Conference on Artificial Life and Robotics (ICAROB2021), January 21 to 24, 2021 


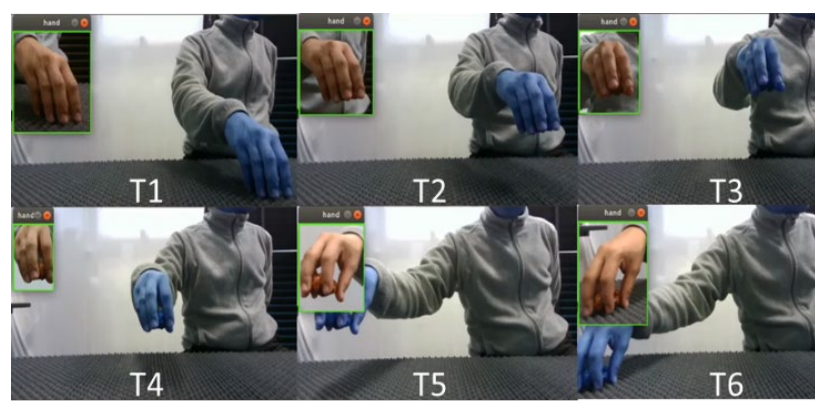

Fig. 2. The Trajectory Data collection procedure. The participant is working on the demonstration by moving his hand for trajectory collection.

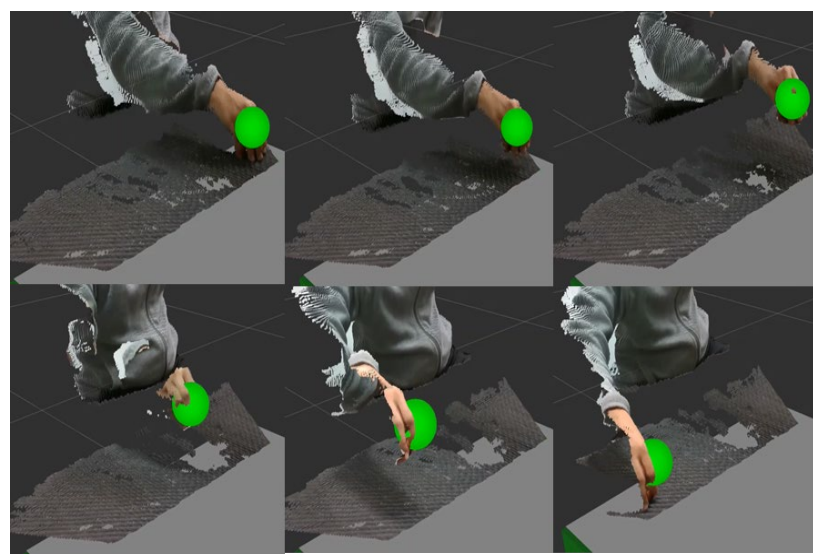

Fig. 3. Human Demonstration in 3-dimension point cloud with hand position detection represented by the green marker.

\section{Experiment and Results}

In our experiment, the user performs the demonstration, which is pick-and-place tasks for food. The robot could detect the hand position in cartesian coordinate then the robot reproduces the motion following the user's hand trajectory by robot end-effector. Firstly, the robot system performs hand detection, then extract the hand position in $3 \mathrm{D}$ cartesian coordinate and record the user's hand trajectory. After that, the robot performs the motion planning and move the end-effector following the trajectory.

\subsection{Human Demonstration by Moving Hand}

The participant makes the motion by his hand in front of the robot. The trajectory of the hand movement follows to the Fig. 2, which the participant moves from right side of the robot then move up. Additionally, Fig. 3 presents the marker of the hand represent in $3 \mathrm{D}$ cartesian coordinate. After that, the user moves the hand down a little from the centre of the trajectory. Finally, the user moves the hand down to the target to the left side of the robot. In hand position estimation, we determine the position using colour image then we map the colour image to depth image to get the cartesian coordinate of hand position. Finally, the trajectory is concatenated by the point to be the waypoint of hand movement. The example of the hand trajectory is shown in Fig 4, which is represented by the marker.

\subsection{Robot Repeating the Human Hand Motion}

Since the participant made the demonstration of hand motion using grasping the object, which is the chicken fry. Since our research would like to develop the robot to assist the worker in the food assembly industry, the participant picks and places the chicken fry from starting point to the target point to teach the robot move to the same motion and target as in Fig. 4 which shows the desired trajectory. After we finish the desired trajectory, the robot moves following the trajectory. In Fig 5, the robot starts from $\mathrm{T} 1$, which the robot moves to the starting position. At T2, the robot moves up to the left side of the robot. At T3, the robot moves the end-effector slightly down as the human demonstration. At T4, the robot moves up to the left side of robot to the target chicken fry. At T5, the robot is approaching to the target to the left side of it. Finally, at T6, the robot could move to the target as the human demonstration before at the same place that hand placed the chicken fry.

\section{Conclusion}

In this paper, to fill the gap of the related research, we present and implement the robot framework of robot motion generation by learning from human hand demonstration and generalize the motion applying to industrial manipulator. It aims toward natural teaching process relating to human learning from experience such as learning from human hand motion.

In this paper, we contribute the method of hand trajectory extraction using deep learning based which is single shot detection to detection hand position. The hand detection using deep neural networks could perform in real-time hand position estimation using RGBD camera to collect 


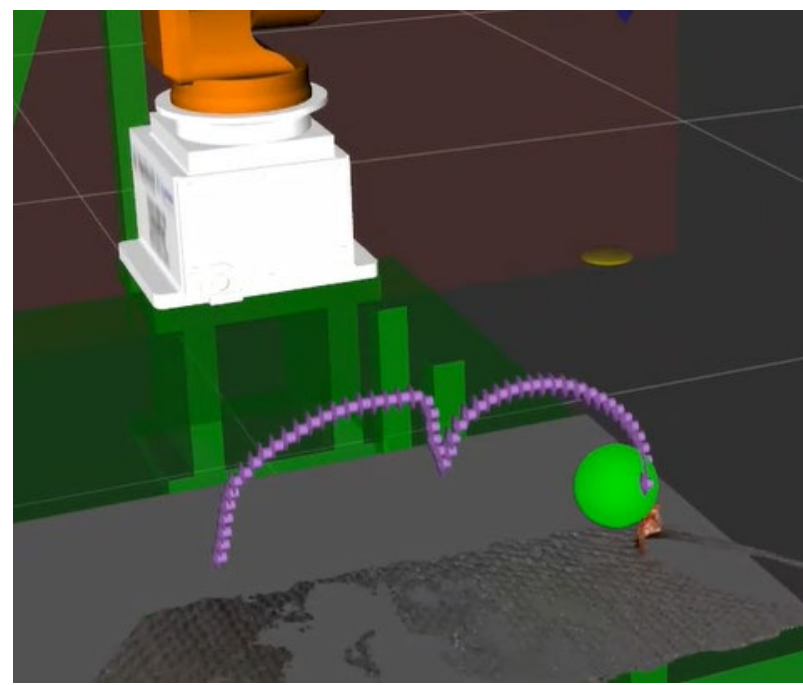

Fig. 4. The overview of the Robot Motion Generation by Hand Demonstration.

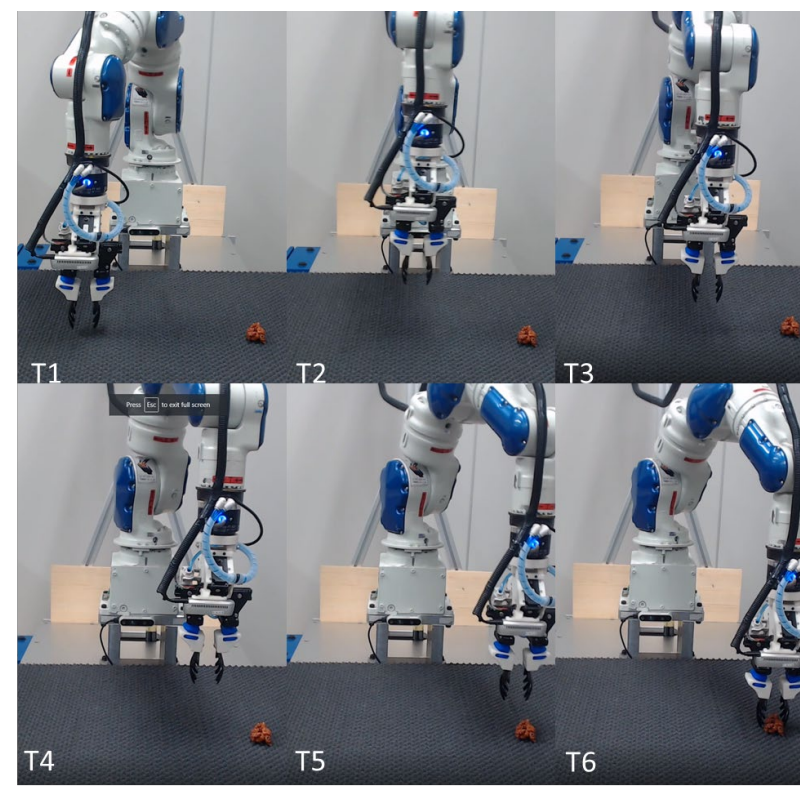

Fig. 5. The capture images of robot motion capture according to the human demonstration.

the trajectory path of hand from the frame by frame from human demonstrating. The second, we proposed the motion generalize motion from hand and generate the motion to the robot. Our approach can generate and generalized motion from hand demonstration without a marker, making the system more reliable and convenient from the real-world tasks. We also successfully experiment and evaluate the performance of the approach.
In future works, we would like to implement with constrain workspace and improve the pose estimation of hand for more reliable learning from humans.

\section{Acknowledgements}

This research was partially supported by the Religion revitalization project in Kitakyushu by the Cabinet Office of Japan.

\section{References}

1. Beeson, Patrick, and Barrett Ames. TRAC-IK: An opensource library for improved solving of generic inverse kinematics. In 2015 IEEE-RAS 15th International Conference on Humanoid Robots (Humanoids), pp. 928935. IEEE, 2015.

2. Sucan, I.A., Moll, M. and Kavraki, L.E., 2012. The open motion planning library. IEEE Robotics \& Automation Magazine, 19(4), pp.72-82.

3. Wang, Weitian, Rui Li, Yi Chen, and Yunyi Jia. "Human intention prediction in human-robot collaborative tasks." In Companion of the 2018 ACM/IEEE international conference on human-robot interaction, pp. 279-280. 2018

4. Jha, Abhishek, and Shital S. Chiddarwar. "Robot programming by demonstration using teleoperation through imitation." Industrial Robot: An International Journal (2017).

5. Schaal, Stefan, Jan Peters, Jun Nakanishi, and Auke Ijspeert. "Learning movement primitives." In Robotics research. the eleventh international symposium, pp. 561572. Springer, Berlin, Heidelberg, 2005.

6. Liu, Wei, Dragomir Anguelov, Dumitru Erhan, Christian Szegedy, Scott Reed, Cheng-Yang Fu, and Alexander C. Berg. "SSD: Single shot multibox detector." In European conference on computer vision, pp. 21-37. Springer, Cham, 2016

(C) The 2021 International Conference on Artificial Life and Robotics (ICAROB2021), January 21 to 24, 2021 